poultry slaughterers. No correlation was found between the results obtained in the laying-hens and those obtained in the other populations. The 27 gentamicin-resistant isolates all showed high-level resistance to gentamicin and two of these isolates, both Enterococcus faecium, were resistant to all antibiotics tested, except vancomycin. The 73 vancomycin-resistant enterococci (VRE) isolates from the five populations belonged to four different species and in all isolates the vanA gene cluster was detected by blot hybridization. The pulsed-field gel electrophoresis (PFGE) patterns of these VRE were quite heterogeneous, but $E$. hirae isolates with the same or a closely related PFGE pattern were isolated at two farms from the broiler farmer and from broilers.

Molecular characterization of vanA-containing transposons of these isolates showed that similar transposon types, predominantly found in poultry, were present. Moreover, similar vanA elements were found not only in isolates with the same PFGE pattern, but also in other VRE isolated from both humans and chickens.

The results of this study suggest transmission of resistance in enterococci from animals to humans. For VRE this might be clonal transmission of animal strains, but transposon transfer seems to occur more commonly.

FROM: van Den Bogaard AE, Willems R, London N, Top J, Stobberingh EE. Antibiotic resistance of faecal enterococci in poultry, poultry farmers and poultry slaughterers. J Antimicrob Chemother 2002;49:497-505.

\section{Targeted Educational Program Reduces Catheter-Related Bloodstream Infections}

Coopersmith and colleagues from the Washington University School of Medicine, St. Louis, reported the results of a study to determine whether an education initiative aimed at improving central venous catheter insertion and care could decrease the rate of primary bloodstream infections.

This was a preintervention and postintervention observational study conducted in an 18-bed surgicalburn-trauma intensive care unit (ICU) in an urban teaching hospital. A total of 4,283 patients were admitted to the ICU between January 1, 1998, and December 31, 2000. A program primarily directed toward registered nurses was developed by a multidisciplinary task force to highlight correct practice for central venous catheter insertion and maintenance. The program consisted of a 10-page selfstudy module on risk factors and practice modifications involved in catheter-related infections, as well as a verbal inservice at staff meetings. Each participant was required to take a pretest before taking the study module and an identical test after its completion. Fact sheets and posters reinforcing the information in the study module were also posted throughout the ICU.

Seventy-four primary bloodstream infections occurred in 6,874 catheter-days (10.8 per 1,000 catheterdays) in the 18 months before the intervention. After the implementation of the education module, the number of primary bloodstream infections fell to 26 in 7,044 catheter- days (3.7 per 1,000 catheter-days), a decrease of $66 \%$ ( $P<$ $.0001)$. The estimated cost savings secondary to the decreased infection rate for the 18 months after the intervention was between $\$ 185,000$ and $\$ 2.808$ million.

The authors concluded that a focused intervention primarily directed at the ICU nursing staff can lead to a dramatic decrease in the incidence of primary bloodstream infections. Educational programs may lead to a substantial decrease in cost, morbidity, and mortality attributable to central venous catheterization.

FROM: Coopersmith CM, Rebmann TL, Zack JE, et al. Effect of an education program on decreasing catheterrelated bloodstream infections in the surgical intensive care unit. Crit Care Med 2002;30:59-64.

\section{CDC Links Infections to Contaminated Allografts}

According to the Centers for Disease Control and Prevention (CDC), as of March 1, 2002, 26 cases of bacterial infections associated with musculoskeletal tissue allografts, including one fatal case, had been reported. Of the 26 cases, 13 patients were infected with Clostridium sordellii, with 11 of these patients receiving tissue from the same tissue processor. None of the tissue transplants in these 26 cases had undergone sterilization. Most tissue transplants only undergo aseptic processing, which does not render them sterile. One reason is that some sterilization technologies can weaken tissue. The CDC also noted that the extended intervals between death and tissue harvesting from the donors likely contributed to the contamination.

The CDC recommended that a method that can kill bacterial spores should be used to process tissue. Unless a sporicidal method is used, aseptically processed tissue should not be considered sterile and healthcare providers should be informed of the possible risk for bacterial infection. If a sporicidal method is unavailable, then tissues should undergo culture and be discarded if Clostridium species or other bacteria normally found in bowel flora are detected. Cultures should also be validated to eliminate false-negatives.

The Food and Drug Administration also updated its industry guidance procedures for processing tissue transplants.

FROM: Centers for Disease Control and Prevention. Update: allograft-associated bacterial infections-United States, 2002. MMWR 2002;51:207-210.

\section{Three-Year Study on Aspergillus in a Hospital Water System}

Nosocomial aspergillosis, a life-threatening infection in immunocompromised patients, is thought to be caused primarily by Aspergillus organisms in the air. Anaissie and colleagues from the University of Arkansas for Medical Sciences, Little Rock, conducted a 3-year prospective study 
of the air, environmental surfaces, and water distribution system of a hospital that had known cases of aspergillosis. The purpose of the study was to determine other possible sources of infection.

Aspergillus species were found in the hospital water system. Significantly higher concentrations of airborne Aspergillus propagules were found in bathrooms, where water use was highest (2.95 colony-forming units $\left.[\mathrm{CFU}] / \mathrm{m}^{3}\right)$, than in patient rooms $\left(0.78 \mathrm{CFU} / \mathrm{m}^{3} ; P=.05\right)$ and in hallways $\left(0.61 \mathrm{CFU} / \mathrm{m}^{3} ; P=.03\right)$. A correlation was found between the rank orders of Aspergillus species recovered from hospital water and air. Water from tanks yielded higher counts of colony-forming units than did municipal water. An isolate of $A$. fumigatus recovered from a patient with aspergillosis was genotypically identical to an isolate recovered from the shower wall in the patient's room.

The authors concluded that in addition to the air, hospital water systems may be a source of nosocomial aspergillosis.

FROM: Anaissie EJ, Stratton SL, Dignani MC, et al. Pathogenic Aspergillus species recovered from a hospital water system: a 3-year prospective study. Clin Infect Dis 2002;34:780-789.

\section{Three Consecutive Outbreaks of Serratia marcescens in a Neonatal Intensive Care Unit}

Fleisch and colleagues from the University Hospital of Zurich, Switzerland, investigated an outbreak of Serratia marcescens in the neonatal intensive care unit (NICU) of this institution. S. marcescens infection was detected in four children transferred from the NICU to the University Children's Hospital (Zurich). All isolates showed identical banding patterns by pulsed-field gel electrophoresis. In a prevalence survey, 11 of 20 neonates were found to be colonized. $S$. marcescens was isolated from bottles of liquid theophylline. Despite replacement of these bottles, $S$. marcescens colonization was detected in additional patients.

Prospective collection of stool and gastric aspirate specimens revealed that colonization occurred in some infants within 24 hours after delivery. These isolates showed a different genotype. Cultures of milk from used milk bottles yielded $S$. marcescens. These isolates showed a third genotype. The method of reprocessing bottles was changed to thermal disinfection. In follow-up prevalence studies, 0 of 29 neonates were found to be colonized by $S$. marcescens.
In summary, three consecutive outbreaks caused by three genetically unrelated clones of $S$. marcescens were documented. Contaminated milk could be identified as the source of at least the third outbreak.

FROM: Fleisch F, Zimmermann-Baer U, Zbinden R, et al. Three consecutive outbreaks of Serratia marcescens in a neonatal intensive care unit. Clin Infect Dis 2002;34:767-773.

\section{MRSA Epidemic Clone in Argentina Involved in Nosocomial Infections}

Infections caused by methicillin-resistant Staphylococcus aureus (MRSA) are a major health concern worldwide for hospitalized patients. In addition, the incidence of community-acquired infection has risen continuously during the past few years. Some MRSA clones spread easier than others within the hospital environment and therefore are frequently implicated in outbreaks. Thus, the spread of a unique epidemic multiresistant clone, the so-called South American clone, is the main cause of nosocomial infections produced by this bacterium in Brazil and in some regions of Argentina, Chile, and Uruguay. Sola and colleagues from the Instituto de Salud Carlos III, Centro Nacional de Microbiologia, Madrid, Spain, describe the identification of a novel clone of MRSA that is involved in nosocomial infections and that shows a prevalence as high as that of the South American clone.

A total of 53 consecutive single-patient MRSA isolates were recovered during a 3-month period (May to July 1999) from six different hospitals ( 955 beds) in Cordoba. The isolates were initially typed according to the antibiotic resistance and phage susceptibility patterns, followed by genotyping using pulsed-field gel electrophoresis (PFGE). PFGE analysis of the 53 MRSA isolates revealed six major types (A to $\mathrm{F}$ ) and 25 subtypes. The B-type DNA pattern was indistinguishable from that of the South American epidemic clone observed in $34 \%$ of the isolates. A novel highly prevalent clone, showing the A-type DNA pattern and representing $38 \%$ of the isolates, was also identified. Moreover, the most frequent subtype of the A clonal family triggered an outbreak in a hospital 2 months later, further confirming its epidemic feature.

FROM: Sola C, Gribaudo G, Vindel A, Patrito L, Bocco JL. Identification of a novel methicillin-resistant Staphylococcus aureus epidemic clone in Cordoba, Argentina, involved in nosocomial infections. J Clin Microbiol 2002;40:1427-1435. 